\title{
Collective modes and quasiparticle interference on the local density of states of cuprate superconductors
}

\author{
C.-T. Chen and N.-C. Yeh \\ Department of Physics, California Institute of Technology, Pasadena, California 91125, USA
}

(Received 24 July 2003; published 23 December 2003)

\begin{abstract}
The energy, momentum, and temperature dependence of the quasiparticle local density of states (LDOS) of a two-dimensional $d_{x^{2}-y^{2}}$-wave superconductor with random disorder is investigated using the first-order $T$-matrix approximation. The results suggest that collective modes such as spin-charge-density waves are relevant low-energy excitations of the cuprates that contribute to the observed LDOS modulations in recent scanning tunneling microscopy studies of $\mathrm{Bi}_{2} \mathrm{Sr}_{2} \mathrm{CaCu}_{2} \mathrm{O}_{x}$.
\end{abstract}

DOI: 10.1103/PhysRevB.68.220505

PACS number(s): 74.72.-h, 74.20.-z, 74.62.Dh, 74.25.Jb

One of the most widely debated issues in cuprate superconductivity is the possibility of preformed Cooper pairs ${ }^{1-4}$ and the origin of the pseudogap phenomenon. ${ }^{5,6}$ Recent experiments have demonstrated that the pseudogap phenomenon is unique to the hole-doped ( $p$-type) cuprates and is absent above $T_{c}$ in electron-doped ( $n$-type) cuprates. ${ }^{7-9}$ Furthermore, in the quasiparticle tunneling spectra of the double-layer $\mathrm{Bi}_{2} \mathrm{Sr}_{2} \mathrm{CaCu}_{2} \mathrm{O}_{8+\delta}(\mathrm{Bi}-2212)$ (Ref. 10) and the one-layer $\mathrm{Bi}_{2}\left(\mathrm{Sr}_{2-x} \mathrm{La}_{x}\right) \mathrm{Cu}_{2} \mathrm{O}_{6+\delta}(\mathrm{Bi}-2201)$ (Ref. 11) systems, it is shown that the pseudogap can be distinguished from the superconducting gap: the former evolves smoothly with increasing temperature whereas the latter vanishes at $T_{c}$. These phenomena suggest that the pseudogap may be associated with a competing order ${ }^{6,12}$ that coexists with the superconducting phase for $T<T_{c}$ and persists above $T_{c}$ until a pseudogap temperature $T^{*}$. The competing quantum ordered phase ${ }^{13,14}$ can be manifested in the form of collective modes such as charge- and spin-density waves (CDW and SDW) in the superconducting state, as inferred from neutronscattering experiments in a variety of $p$-type cuprates. ${ }^{15-19}$ However, whether these collective modes are closely correlated with superconductivity remain controversial. Recent scanning tunneling spectroscopic (STM) studies of the Fourier transformed (FT) quasiparticle local density of states (LDOS) of Bi-2212 (Refs. 20-22) have stimulated further discussions on the relevance of collective modes. ${ }^{23-28}$ While Bogoliubov quasiparticle interference apparently plays an important role in the observed FT-LDOS in the superconducting state, certain spectral details of the LDOS cannot be accounted for unless collective modes are considered. ${ }^{23-25}$ In particular, the findings of four high-intensity Bragg peaks remaining above $T_{c}$ in the FT-LDOS map of Bi-2212 (Ref. 29) cannot be reconciled with quasiparticles being the sole low-energy excitations. These new developments motivate us to reexamine the role of collective modes in cuprate superconductors by considering the energy $E$, momentum transfer $q$, and temperature $T$ dependence of the resulting FT-LDOS modulations.

We begin our model construction by noting that substantial quasiparticle gap inhomogeneities are observed in the low-temperature tunneling spectroscopy of underdoped and optimally doped Bi-2212 single crystals, ${ }^{30,31}$ suggesting at least two types of spatially separated regions, one with sharp quasiparticle coherence peaks at smaller energies $\Delta_{d}$ and the other with rounded hump-like features at larger energies $\Delta^{*}$. On the other hand, low-energy LDOS (for $E<0.5 \Delta_{d}$ ) of Bi2212 exhibit long-range spectral homogeneity. We therefore conjecture that dynamic SDW or CDW coexist with cuprate superconductivity and that they are only manifested in the quasiparticle LDOS when pinned by disorder. Thus, regions with rounded hump features in the quasiparticle spectra are manifestation of localized charge modulations due to pinning of collective modes by disorder, and the wave vector of the charge modulation is twice of that for the collinear SDW order, as proposed in Refs. 23,24 and 32. In contrast, regions with sharp quasiparticle spectral peaks are representative of generic Bogoliubov quasiparticle spectra with a well-defined $d$-wave pairing order parameter $\Delta_{\mathbf{k}} \approx \Delta_{d} \cos 2 \theta_{\mathbf{k}}$, where $\Delta_{d}$ is the maximum gap value and $\theta_{\mathbf{k}}$ is the angle between the quasiparticle wave vector $\mathbf{k}$ and the antinode direction. Our model therefore assumes "puddles" of spatially confined "pseudogap regions" with a quasiparticle scattering potential modulated at a periodicity of four lattice constants along the $\mathrm{Cu}-\mathrm{O}$ bonding directions, and the spatial modulations can be of either the "checkerboard" pattern" ${ }^{23,24}$ or "charge nematic" with short-range stripes. ${ }^{12,14}$ In the limit of weak perturbations, we employ the first-order $T$-matrix approximation and consider a $(400 \times 400)$ sample area with either 24 randomly distributed point impurities or 24 randomly distributed puddles of charge modulations that cover $\approx 6 \%$ of the sample area. For simplicity, we do not consider the effect of disorder on either suppressing the local pairing potential $\Delta_{d}(r)$ or altering the nearest-neighbor hopping coefficient $t$ in the band structure of Bi-2212, although such effects reflect the internal structures of charge modulations. ${ }^{25,26}$

Specifically, the Hamiltonian of the two-dimensional superconductor is given by $\mathcal{H}=\mathcal{H}_{B C S}+\mathcal{H}_{\text {imp }}$, where $\mathcal{H}_{B C S}$ denotes the unperturbed BCS Hamiltonian of the $d$-wave superconductor, $\mathcal{H}_{B C S}=\Sigma_{\mathbf{k} \sigma}\left(\epsilon_{k}-\mu\right) c_{\mathbf{k} \sigma}^{\dagger} c_{\mathbf{k} \sigma}+\Sigma_{\mathbf{k}} \Delta_{\mathbf{k}}\left[c_{\mathbf{k} \uparrow}^{\dagger} c_{-\mathbf{k} \downarrow}^{\dagger}\right.$ $\left.+c_{-\mathbf{k} \downarrow} c_{\mathbf{k} \uparrow}\right]$, and $\mathcal{H}_{\text {imp }}$ is the perturbation Hamiltonian associated with impurity-induced quasiparticle scattering potential. $^{24,26,27,33}$ Using the T-matrix method, the Green's function $\mathcal{G}$ associated with $\mathcal{H}$ is given by $\mathcal{G}=\mathcal{G}_{0}+\mathcal{G}_{0} T \mathcal{G}_{0}$, where $\mathcal{G}_{0}$ is the Green's function of $\mathcal{H}_{B C S}$ and $T=\mathcal{H}_{\text {imp }} /(1$ $\left.-\mathcal{G}_{0} \mathcal{H}_{\text {imp }}\right)$. The Hartree perturbation potential for single scattering events in the diagonal part of $\mathcal{H}$ and for noninter- 
acting identical point impurities at locations $\mathbf{r}_{i}$ is $V_{\alpha}(\mathbf{q})$ $=\sum_{i} V_{s, m} e^{i \mathbf{q} \cdot \mathbf{r}_{i}}$ for nonmagnetic $\left(V_{s}\right)$ and magnetic $\left(V_{m}\right)$ impurities, ${ }^{27}$ whereas that for puddles with short stripelike modulations centering at $\mathbf{r}_{j}$ is ${ }^{33}$

$$
V_{\beta}(\mathbf{q})=\sum_{j} V_{0} e^{i \mathbf{q} \cdot \mathbf{r}_{j}} \frac{2 \sin \left(q_{y, x} R_{j}\right) \sin \left(q_{x, y} R_{j}\right)}{q_{y, x} \sin \left(2 q_{x, y}\right)},
$$

and that for checkerboard modulations is

$$
V_{\gamma}(\mathbf{q})=\sum_{j} V_{0} e^{i \mathbf{q} \cdot \mathbf{r}_{j}}\left[\frac{2 \sin \left(q_{y} R_{j}\right) \sin \left(q_{x} R_{j}\right)}{q_{y} \sin \left(2 q_{x}\right)}+\left(q_{x} \leftrightarrow q_{y}\right)\right] .
$$

Here all lengths are expressed in units of the lattice constant $a, R_{j}$ is the averaged radius of the $j$ th puddle, and $V_{0}$ denotes the magnitude of the scattering potential by pinned collective modes. For simplicity, we neglect the energy dependence of $V_{\alpha, \beta, \gamma}$ and assume that $V_{s}, V_{m}$, and $V_{0}$ are sufficiently small so that no resonance occurs in the FT-LDOS. ${ }^{26}$ For sufficiently large scattering potentials, full $T$-matrix calculations become necessary as in Ref. 27. However, large $V_{s, m}$ would result in strong spectral asymmetry between positive and negative bias voltages, ${ }^{27}$ which differs from experimental observation. ${ }^{20-22}$ We also note that the energy dependence of $V_{\beta, \gamma}$ reflects the spectral characteristics of the collective modes and their interaction with quasiparticles and impurities. For instance, we expect $V_{\gamma} \sim \zeta \gamma^{2}$ for pinned SDW, where $\zeta$ is the impurity pinning strength and $\gamma$ is the coupling amplitude of quasiparticles with SDW fluctuations. ${ }^{23,24}$ Empirically for nearly optimally doped $\mathrm{Bi}-2212, R_{j}$ ranges from $5-10 .{ }^{31}$ Here we take different values for $R_{j}$ with a mean value $\left\langle R_{j}\right\rangle=10$.

Given the Hamiltonian and the scattering potentials $V_{\alpha, \beta, \gamma}(\mathbf{q})$, we find that for infinite quasiparticle lifetime and in the first-order $T$-matrix approximation, the FT of the LDOS $\rho(\mathbf{r}, E)$ that involves elastic scattering of quasiparticles from momentum $\mathbf{k}$ to $\mathbf{k}+\mathbf{q}$ is

$$
\begin{aligned}
\rho_{\mathbf{q}}(\omega)= & -\frac{1}{\pi N^{2}} \lim _{\delta \rightarrow 0} \sum_{\mathbf{k}} V_{\alpha, \beta, \gamma}(\mathbf{q})\left\{u _ { \mathbf { k } + \mathbf { q } } u _ { \mathbf { k } } \left(u_{\mathbf{k}+\mathbf{q}} u_{\mathbf{k}}\right.\right. \\
& \left.\mp v_{\mathbf{k}+\mathbf{q}} v_{\mathbf{k}}\right) \operatorname{Im}\left[\frac{1}{\left(\omega-E_{\mathbf{k}}+i \delta\right)\left(\omega-E_{\mathbf{k}+\mathbf{q}}+i \delta\right)}\right] \\
& +u_{\mathbf{k}+\mathbf{q}} v_{\mathbf{k}}\left(u_{\mathbf{k}+\mathbf{q}} v_{\mathbf{k}}\right. \\
& \left. \pm v_{\mathbf{k}+\mathbf{q}} u_{\mathbf{k}}\right) \operatorname{Im}\left[\frac{1}{\left(\omega+E_{\mathbf{k}}+i \delta\right)\left(\omega-E_{\mathbf{k}+\mathbf{q}}+i \delta\right)}\right] \\
& +v_{\mathbf{k}+\mathbf{q}} u_{\mathbf{k}}\left(u_{\mathbf{k}+\mathbf{q}} v_{\mathbf{k}}\right. \\
& \left. \pm v_{\mathbf{k}+\mathbf{q}} u_{\mathbf{k}}\right) \operatorname{Im}\left[\frac{1}{\left(\omega-E_{\mathbf{k}}+i \delta\right)\left(\omega+E_{\mathbf{k}+\mathbf{q}}+i \delta\right)}\right] \\
& -v_{\mathbf{k}+\mathbf{q}} v_{\mathbf{k}}\left(u_{\mathbf{k}+\mathbf{q}} u_{\mathbf{k}}\right. \\
& \left.\left.\mp v_{\mathbf{k}+\mathbf{q}} v_{\mathbf{k}}\right) \operatorname{Im}\left[\frac{1}{\left(\omega+E_{\mathbf{k}}+i \delta\right)\left(\omega+E_{\mathbf{k}+\mathbf{q}}+i \delta\right)}\right]\right\} .
\end{aligned}
$$

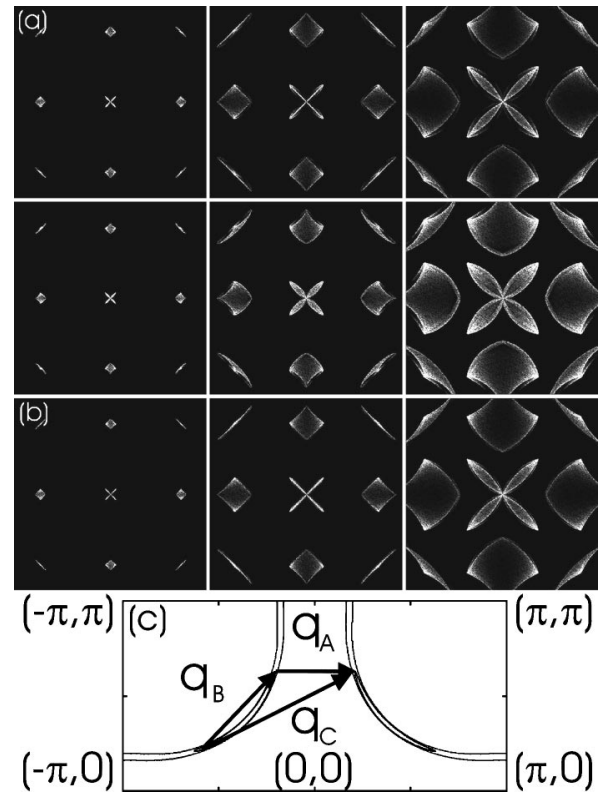

FIG. 1. Calculated energy-dependent Fourier transform (FT) maps of quasiparticle LDOS in the first Brillouin zone with randomly distributed nonmagnetic point defects using Eq. (3) and $V_{\alpha}$ : (a) $\Delta_{d}=40 \mathrm{meV}$ and $\left(\omega / \Delta_{d}\right)= \pm 0.15, \pm 0.45, \pm 0.75$ (up and down from left to right); (b) $\Delta_{d}=20 \mathrm{meV}$ and $\left(\omega / \Delta_{d}\right)=0.15,0.45,0.75$ (left to right). (c) Schematic illustration of the equal-energy contours and representative modulation wave vectors $\mathbf{q}_{A}, \mathbf{q}_{B}$ and $\mathbf{q}_{C}$, which correspond to $\mathbf{q}_{1}, \mathbf{q}_{7}$, and $\mathbf{q}_{2}$ in Refs. 20,21.

Here $N$ is the total number of unit cells in the sample and $\operatorname{Im}[\cdots]$ denotes the imaginary part of the quantity within the brackets, which is related to the equal-energy quasiparticle joint density of states. The upper (lower) sign in the coherence factor applies to spin-independent (spindependent) interactions, $u_{\mathbf{k}}$ and $v_{\mathbf{k}}$ are the Bogoliubov quasiparticle coefficients, $u_{\mathbf{k}}^{2}+v_{\mathbf{k}}^{2}=1, u_{\mathbf{k}}^{2}=\left[1+\left(\xi_{\mathbf{k}} / E_{\mathbf{k}}\right)\right] / 2, \quad \xi_{\mathbf{k}}$ $\equiv \epsilon_{\mathrm{k}}-\mu, \epsilon_{\mathrm{k}}$ is the tight-binding energy of the normal state of Bi-2212 according to Norman et al., ${ }^{34}$

$$
\begin{aligned}
\epsilon_{\mathbf{k}}= & t_{1}\left(\cos k_{x}+\cos k_{y}\right) / 2+t_{2} \cos k_{x} \cos k_{y}+t_{3}\left(\cos 2 k_{x}\right. \\
& \left.+\cos 2 k_{y}\right) / 2+t_{4}\left(\cos 2 k_{x} \cos k_{y}+\cos k_{x} \cos 2 k_{y}\right) / 2 \\
& +t_{5} \cos 2 k_{x} \cos 2 k_{y},
\end{aligned}
$$

where $t_{1-5}=-0.5951,0.1636,-0.0519,-0.1117,0.0510$ $\mathrm{eV}, \mu$ is the chemical potential, and $E_{\mathbf{k}}=\sqrt{\xi_{\mathbf{k}}^{2}+\Delta_{\mathbf{k}}^{2}}$.

Using Eq. (3) and $V_{\alpha, \beta, \gamma}(\mathbf{q})$, we obtain the energydependent FT-LDOS maps in the first Brillouin zone for nonmagnetic point impurities in Fig. 1 with two different $\Delta_{d}$ values and for pinned SDW (with spin-dependent coherence factor) in Fig. 2, whereas the corresponding LDOS modulations due to $V_{\alpha, \beta, \gamma}(\mathbf{q})$ in real space is shown in Figs. 3(a)3(c). For nonmagnetic point impurity scattering at $T \ll T_{c}$, the intensities associated with $\mathbf{q}_{B}$ and $\mathbf{q}_{C}$ are much stronger than those of $\mathbf{q}_{A}$, as shown in Fig. 1 and also in Fig. 4(a). The results in Fig. 1 differ from the STM observation ${ }^{20,21}$ that reveals comparable intensities associated with $\mathbf{q}_{A}$ and $\mathbf{q}_{B}$, and weaker intensities with $\mathbf{q}_{C}$. Interestingly, the intensities of $\mathbf{q}_{A}$ and $\mathbf{q}_{B, C}$ become reversed if one assumes mag- 

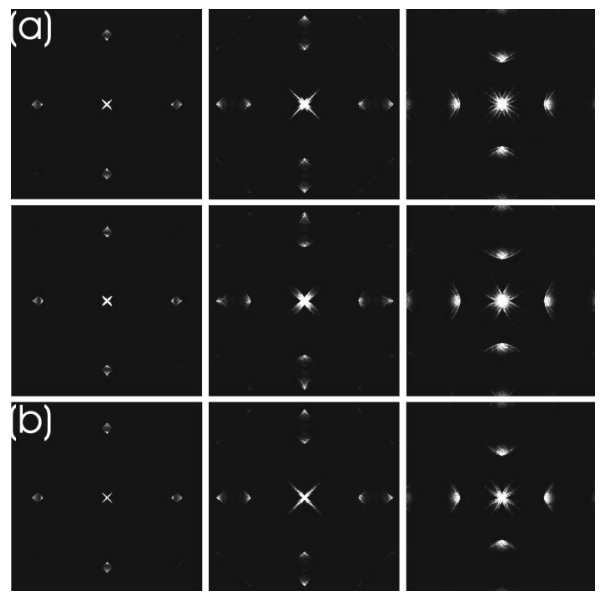

FIG. 2. Energy-dependent FT-LDOS maps with randomly distributed pinned SDW using Eq. (3) and $V_{\gamma}$ : (a) $\Delta_{d}=40 \mathrm{meV}$ and $\left(\omega / \Delta_{d}\right)= \pm 0.15, \pm 0.45, \pm 75$, up and down from left to right; (b) $\Delta_{d}=20 \mathrm{meV}$ and $\left(\omega / \Delta_{d}\right)=0.15,0.45,0.75$, from left to right. The FT-LDOS does not exhibit discernible differences in the spectral characteristics except the total intensities if we simply replace $V_{\gamma}$ by $V_{\beta}$ and assume nonmagnetic coherence factors in Eq. (3).

netic point impurity scattering, as illustrated in Fig. 4(b). However, there is no evidence of magnetic scattering in the samples used in Refs. 20 and 21. In contrast, the presence of pinned collective modes, regardless of CDW or SDW, gives rise to much stronger intensities for $\mathbf{q}_{A}$ (by about two orders of magnitude), as shown in Fig. 2. Thus, the empirical FTLDOS maps ${ }^{20,21}$ cannot be solely attributed to quasiparticle scattering by nonmagnetic point impurities.

The relevance of collective modes become indisputable when we consider the temperature dependence of the FTLDOS. As shown in Fig. 5(a), in the limit of $T \rightarrow T_{c}^{-}$, the $q$ values contribute to the FT-LDOS map become significantly extended and smeared for point-impurity scattering. In contrast, pinned SDW yields strong intensities in the FT-LDOS map only at $\mathbf{q}_{A}$ for $T>\sim T_{c}$, as shown in Fig. 5(b). The overall energy dispersion due to pinned SDW is weaker than that due to point impurities, as shown in Fig. 5(c) for $\left|\mathbf{q}_{A}\right|$-vs- $V$ (biased voltage) at both $T=0$ and $T=T_{c}$. In particular, we note that the dispersion is further reduced at $T_{c}$. These findings are consistent with recent experimental observation by Yazdani et al. $^{29}$

The energy, momentum, and temperature dependence of our calculated FT-LDOS in Figs. 1-5 is supportive of spatially modulated collective modes being relevant low-energy
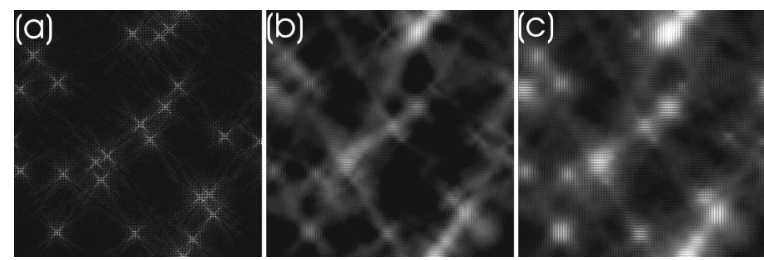

FIG. 3. Real-space quasiparticle LDOS for a $(400 \times 400)$ area at $T=0$ due to scattering by (a) nonmagnetic point impurities, (b) pinned $\mathrm{CDW}$, and (c) pinned SDW for $\Delta_{d}=40 \mathrm{meV}$ and $\omega$ $=30 \mathrm{meV}$.

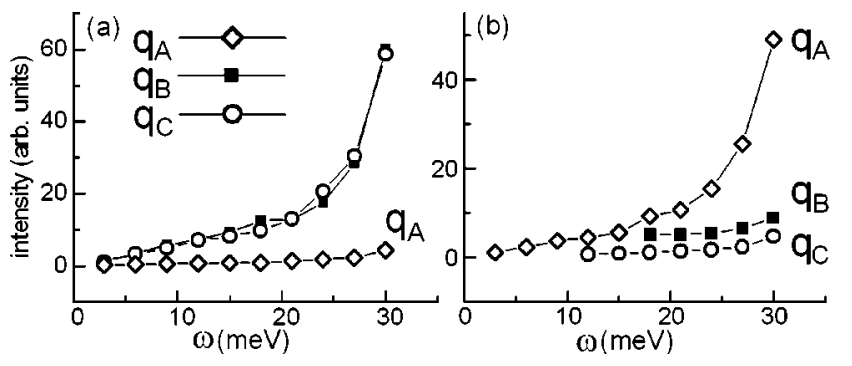

FIG. 4. Evolution of the relative intensities of FT-LDOS with energy $(\omega)$ for $q_{A}, q_{B}$, and $q_{C}$ as defined in Fig. 1(c) and $V_{s}, V_{m}$, and $V_{0}$ all taken to be unity: quasiparticle scattering by (a) single nonmagnetic point impurity and (b) single magnetic point impurity.

excitations in cuprates besides quasiparticles. In particular, only pinned collective modes can account for the observation in the FT-LDOS map above $T_{c}$. Although our simplified model cannot exclude CDW, we note that pinned CDW would have coupled directly to the quasiparticle spectra and resulted in stripelike periodic local conductance modulation, which has not been observed in STM studies. On the other hand, various puzzling phenomena seem reconcilable with the SDW scenario. For instance, the nanoscale gap variations observed in Bi-2212 (Ref. 31) may be understood by noting that the LDOS in regions with disorder-pinned SDW con-
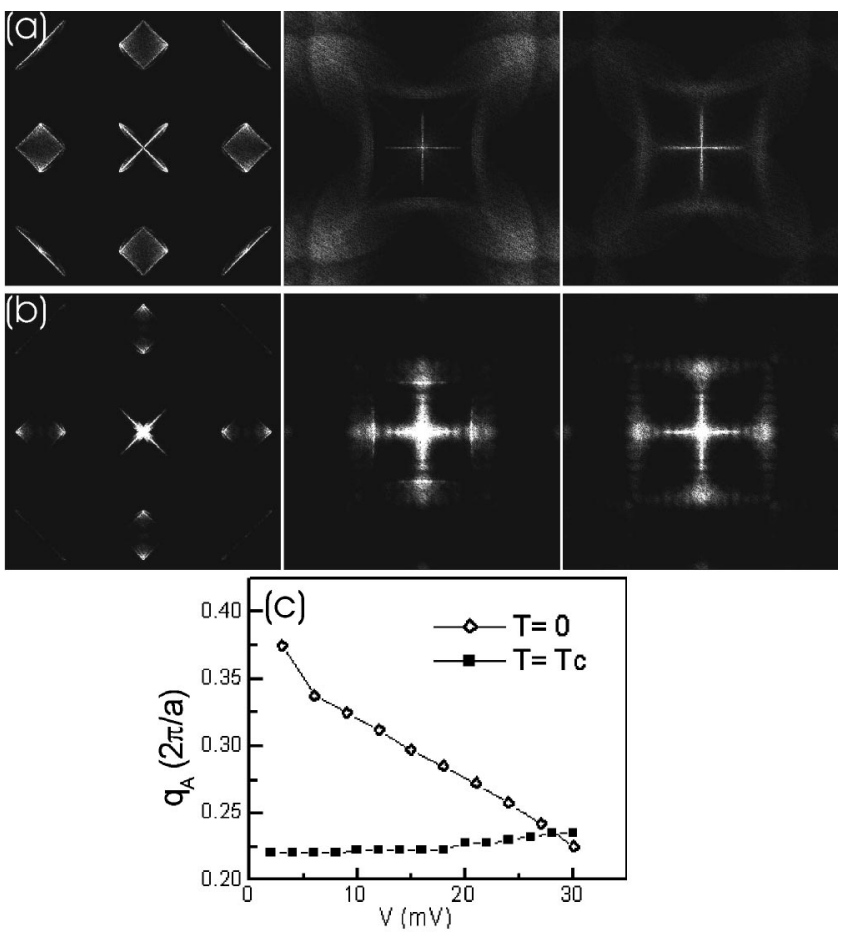

FIG. 5. The FT-LDOS maps at $T=0,0.75 T_{c}$, and $T_{c}$ (from left to right) for (a) point impurities $V_{\alpha}(q)$ and (b) pinned SDW $V_{\gamma}(q)$. We assume $\Delta_{d}(T)=\Delta_{d}(0)\left[1-\left(T / T_{c}\right)\right]^{1 / 2}, \Delta_{d}(0)=40 \mathrm{meV}$, tunneling biased voltage $=18 \mathrm{mV}$, and $T_{c}=80 \mathrm{~K}$. Besides temperature-dependent coherence factors, the thermal smearing of quasiparticle tunneling conductance $(d I / d V)$ is obtained by using $(d I / d V) \propto\left|\int \rho_{\mathbf{q}}(E)(d f / d E)\right|_{(E-e V)} d E \mid$, where $f(E)$ denotes the Fermi function. (c) $\left|q_{A}\right|$-vs- $V$ (biased voltage) dispersion relation for pinned SDW at $T=0$ and $T_{c}$. 
tains information of disorder potential coupled with quasiparticles and SDW, so that the humplike spectral features at $\pm \Delta^{*}$ represent neither the SDW gap nor the superconducting gap $\Delta_{d}$, and the values of $\Delta^{*}$ vary in accordance with the disorder potential. The long-range spatial homogeneity of quasiparticle spectra in $\mathrm{YBa}_{2} \mathrm{Cu}_{3} \mathrm{O}_{7-\delta}$ (YBCO) (Ref. 35) as opposed to the strong spatial inhomogeneity in Bi-2212 can also be reconciled in a similar context. That is, SDW can be much better pinned in extreme two-dimensional cuprates like Bi-2212 than in more three-dimensional cuprates such as YBCO. Furthermore, SDW can be stabilized by magnetic fields, ${ }^{23,24,36}$ which naturally account for the checkerboardlike spectral modulations around the vortex cores of Bi-2212. ${ }^{19,37}$ Finally, the smooth evolution of the pseudogap phase with temperature through $T_{c}$ may contribute to the anomalously large Nernst effect under a $c$ axis magnetic field above $T_{c},{ }^{38}$ with spin fluctuations responsible for the excess entropy.

In summary, we employ first-order $T$-matrix approximation to study modulations in the quasiparticle FT-LDOS of cuprates as a function of energy, momentum, and temperature. Our results suggest that a full account for all aspects of experimental observation below $T_{c}$ must include collective modes as relevant low-energy excitations besides quasiparticles, and that only collective modes can account for the observed FT-LDOS above $T_{c}$.

We thank Professors Subir Sachdev, Doug Scalapino, C. S. Ting, and Mr. Yuan-Yu Jau for useful discussions. This research was supported by NSF Grant No. DMR-0103045.

${ }^{1}$ P.W. Anderson, Science 235, 1196 (1987).

${ }^{2}$ E. Dagotto, J. Riera, and D. Scalapino, Phys. Rev. B 45, 5744 (1992).

${ }^{3}$ V.J. Emery, S.A. Kivelson, and O. Zachar, Phys. Rev. B 56, 6120 (1997).

${ }^{4}$ Q.J. Chen, I. Kosztin, B. Janko, and K. Levin, Phys. Rev. B 59, 7083 (1999).

${ }^{5}$ T. Timusk and B. Statt, Rep. Prog. Phys. 62, 61 (1999).

${ }^{6}$ N.-C. Yeh, Bulletin of Assoc. Asia Pacific Phys. Soc. 12, 2 (2002); cond-mat/0210656.

${ }^{7}$ C.-T. Chen, P. Seneor, N.-C. Yeh, R. Vasquez, L. Bell, C. Jung, J. Kim, M. Park, H. Kim, and S. Lee, Phys. Rev. Lett. 88, 227002 (2002)

${ }^{8}$ S. Kleefisch, B. Welter, A. Marx, L. Alff, R. Gross, and M. Naito, Phys. Rev. B 6310, 100507 (2001).

${ }^{9}$ N.-C. Yeh, C.-T. Chen, R.P. Vasquez, C.U. Jung, S.I. Lee, K. Yoshida, and S. Tajima, J. Low Temp. Phys. 131, 435 (2003).

${ }^{10}$ V.M. Krasnov, A. Yurgens, D. Winkler, P. Delsing, and T. Claeson, Phys. Rev. Lett. 84, 5860 (2000).

${ }^{11}$ A. Yurgens, D. Winkler, T. Claeson, S. Ono, and Y. Ando, Phys. Rev. Lett. 90, 147005 (2003).

${ }^{12}$ N.-C. Yeh and C.-T. Chen, Int. J. Mod. Phys. B 17, 3575 (2003); cond-mat/0302217.

${ }^{13}$ S. Sachdev, Rev. Mod. Phys. 75, 913 (2003).

${ }^{14}$ S.A. Kivelson, I.P. Bindloss, E. Fradkin, V. Oganesyan, J.M. Tranquada, A. Kapitulnik, and C. Howald, Rev. Mod. Phys. 75, 1201 (2003); cond-mat/0210683 (unpublished), and references therein.

${ }^{15}$ M.A. Kastner, R.J. Birgeneau, G. Shirane, and Y. Endoh, Rev. Mod. Phys. 70, 897 (1998), and references therein.

${ }^{16}$ H.F. Fong, P. Bourges, Y. Sidis, L.P. Regnault, A. Ivanov, G. Gu, N. Koshizuka, and B. Keimer, Nature (London) 398, 588 (1999).

${ }^{17}$ H.A. Mook, P.C. Dai, and F. Dogan, Phys. Rev. Lett. 88, 097004 (2002).

${ }^{18}$ M. Fujita, H. Goka, K. Yamada, and M. Matsuda, Phys. Rev. Lett. 88, 167008 (2002).

${ }^{19}$ B. Lake et al., Science 291, 1759 (2001).

${ }^{20}$ J.E. Hoffman, K. McElroy, D.H. Lee, K.M. Lang, H. Eisaki, S. Uchida, and J. Davis, Science 297, 1148 (2002).

${ }^{21}$ K. McElroy, R. Simmonds, J. Hoffman, D. Lee, J. Orenstein, H. Eisaki, S. Uchida, and J. Davis, Nature (London) 422, 592 (2003).

${ }^{22}$ C. Howald, H. Eisaki, N. Kaneko, M. Greven, and A. Kapitulnik, Phys. Rev. B 67, 014533 (2003).

${ }^{23}$ A. Polkovnikov, M. Vojta, and S. Sachdev, Phys. Rev. B 65, 220509 (2002)

${ }^{24}$ A. Polkovnikov, S. Sachdev, and M. Vojta, Physica C 388, 19 (2003).

${ }^{25}$ D. Podolsky, E. Demler, K. Damle, and B.I. Halperin, Phys. Rev. B 67, 094514 (2003).

${ }^{26}$ D.G. Zhang and C.S. Ting, Phys. Rev. B 67, 100506 (2003).

${ }^{27}$ Q.H. Wang and D.H. Lee, Phys. Rev. B 67, 020511 (2003).

${ }^{28}$ L. Capriotti, D.J. Sacalapino, and R.D. Sedgewick, Phys. Rev. B 68, 014508 (2003).

${ }^{29}$ A. Yazdani (private communications).

${ }^{30}$ C. Howald, P. Fournier, and A. Kapitulnik, Phys. Rev. B 64, 100504 (2001).

${ }^{31}$ K.M. Lang, V. Madhavan, J. Hoffman, E. Hudson, H. Eisaki, S. Uchida, and J. Davis, Nature (London) 415, 412 (2002).

${ }^{32}$ O. Zachar, S.A. Kivelson, and V.J. Emery, Phys. Rev. B 57, 1422 (1998).

${ }^{33}$ For coexisting superconductivity and $\mathrm{CDW}, V_{\beta}(\mathbf{q})$ represents the second-order effect of quasiparticle interference with pinned CDW. The first-order effect of CDW has been discussed in Ref. 25.

${ }^{34}$ M.R. Norman, M. Randeria, H. Ding, and J.C. Campuzano, Phys. Rev. B 52, 615 (1995).

${ }^{35}$ N.-C. Yeh et al., Phys. Rev. Lett. 87, 087003 (2001).

${ }^{36}$ E. Demler, S. Sachdev, and Y. Zhang, Phys. Rev. Lett. 87, 067202 (2001).

${ }^{37}$ J.E. Hoffman, E. Hudson, K. Lang, V. Madhavan, H. Eisaki, S. Uchida, and J. Davis, Science 295, 466 (2002).

${ }^{38}$ Y.Y. Wang, N.P. Ong, Z. Xu, T. Kakeshita, S. Uchida, D. Bonn, R. Liang, and W. Hardy, Phys. Rev. Lett. 88, 257003 (2002). 